\section{P113 (continued)}

compared to $20.4 \%$ with medium acculturation and $6.8 \%$ with low acculturation $(P<.009)$.

Conclusions: Findings suggest that Americanized Hispanic adolescents consume more standard American foods and fewer meals prepared at home. Future investigations should explore the impact of acculturation on diet quality among other ethnicities and evaluate culturally appropriate interventions in the US.

Funding: USDA.

\section{Food and Nutrition Policy}

\section{P114 The Potential Impacts of Childhood Experiences of Forced Eating on Vegetable Consumption Among Korean Young Adults}

So-Young Kim, PhD, sonyah@sch.ac.kr, Soonchunhyang University, 22 Soonchunhyang-ro, Asan, Chungnam, South Korea, 31538; Eunju Lee, EdD, Daegok Elementary School

Background: Previous research has highlighted that forced eating might lead to a long-lasting food rejection. Vegetables are the most common subject of forced eating due to their sensory bitterness, causing an instinct-guided rejection. Currently, unbalanced diet with insufficient vegetable consumption is being recognized, particularly among young adults. This problem appears to be associated with multi-dimensional factors but might also be related to experiences of forced eating.

Objective: This study aimed to explore the potential impacts of childhood experiences of forced eating on vegetable consumption among Korean young adults.

Study Design, Setting, Participants: An online survey was conducted from February 1824, 2020 among Korean young adults in their 20s. Out of 1277 responses, $461(36.1 \%)$ responses reporting to have experienced forced eating were analyzed.

Measurable Outcome/Analysis: Descriptive statistics were calculated, and significant differences in vegetable consumption (ie, vegetable preference, vegetable acceptance, and perceived daily intake of vegetables) were measured according to experiences of forced eating.

Results: Forced eating occurred mostly when they were elementary school students (57.2\%), and preschool children (21.2\%). It happened mostly frequently at home $(51.0 \%)$, followed by schools (26.5\%). Respondents mostly identified parents $(50.1 \%)$, followed by school teachers $(26.0 \%)$ as authority figures involved in forced eating. Approximately $41.0 \%$ of them were still not able to eat the target foods of forced eating, and $44.2 \%$ acknowledged its negative impacts on their current dietary habits. Among the target foods of forced eating, vegetables were most prevalent (57.9\%). Vegetable preference, vegetable acceptance, and perceived daily intake of vegetables tended to be significantly lower when they had experiences of forced eating.

Conclusions: This study implies that childhood experiences of forced eating could make negative impacts on future dietary habits related to vegetable consumption. Despite some limitations, it may shed light on the importance and necessity of preventing forced eating during childhood and intervening its negative impacts on dietary habits.

Funding: The National Research Foundation of Korea.

\section{Nutrition Across the Life Cycle}

\section{P115 Breastfeeding Peer Counselors: Support Mothers Can Trust}

Sheilah Hebert, MS, RDN, IBCLC, murphysh@msu.edu, Michigan State University, 5303 South Cedar, Lansing, MI, 48911; Latashia Perry, CLC, Michigan State University

Objective: The Flint water crisis left many mothers of all income levels with limited trust in outside resources. With the support of the Greater Flint Community Foundation, a peer counselor was hired to support breastfeeding mothers. A peer counselor is a local mother representative of the community who has already developed trust within the community. This study looks at the effectiveness of community-funded breastfeeding peer counselors representative of their communities.

Use of Theory or Research: One of the most highly effective preventative measures a mother can take to protect the health of her infant is to breastfeed. In the US, $81 \%$ of babies initiate breastfeeding; $22 \%$ are exclusively breastfed 6 months later. According to the CDC, peer support programs are effective in increasing initiation, duration, and exclusivity of breastfeeding. Peer counseling is rooted in the theory of reasoned action which recognizes the influence of peers on individual behavior. Breastfeeding peer counselors help to normalize breastfeeding within their own community.

Target Audience: Pregnant and breastfeeding women who reside in Flint, Michigan.

Program Description: The peer counselor provided evidence-based education and support to increase initiation, duration, and exclusivity. The peer counselor educated, supported, and encouraged women to continue breastfeeding exclusively for 6 months and continue until at least 1 year of age. The peer counselor conducted home visits and was available during evenings and weekends. The number of visits varied depending on the needs of the mother. If possible, at least 1 visit was completed prenatally and minimally 1 visit within the first week after delivery. Additional contacts were provided through home visits, phone calls and FaceTime during the immediate postpartum period when one-on-one support for initiation of breastfeeding is critical.

Evaluation Methods: Women were recruited through referrals from a variety of community agencies that work with families in the city of Flint. Demographic (age, race, ethnicity, income), initiation, duration, and exclusivity information was collected from all participants who enrolled in program.

Results: Fifty presentations on benefits of breastfeeding reached 547 families between September 2018 - September

Continued on page $S 71$ 